\title{
Some examples of hyperarchimedean lattice-ordered groups
}

by

\author{
Anthony W. Hager (Middletown, CT) and \\ Chawne M. Kimber (Easton, PA)
}

\begin{abstract}
All $\ell$-groups shall be abelian. An $a$-extension of an $\ell$-group is an extension preserving the lattice of ideals; an $\ell$-group with no proper $a$-extension is called $a$-closed. A hyperarchimedean $\ell$-group is one for which each quotient is archimedean. This paper examines hyperarchimedean $\ell$-groups with unit and their $a$-extensions by means of the Yosida representation, focussing on several previously open problems. Paul Conrad asked in 1965: If $G$ is $a$-closed and $M$ is an ideal, is $G / M a$-closed? And in 1972: If $G$ is a hyperarchimedean sub- $\ell$-group of a product of reals, is the $f$-ring which $G$ generates also hyperarchimedean? Marlow Anderson and Conrad asked in 1978 (refining the first question above): If $G$ is $a$-closed and $M$ is a minimal prime, is $G / M a$-closed? If $G$ is $a$-closed and hyperarchimedean and $M$ is a prime, is $G / M$ isomorphic to the reals? Here, we introduce some techniques of $a$-extension and construct a several parameter family of examples. Adjusting the parameters provides answers "No" to the questions above.
\end{abstract}

1. Preliminaries. In the following, a lattice-ordered group, or an $\ell$ group, is an abelian group $(G,+)$ with a lattice order $\leq$ for which $a \leq b$ implies $a+c \leq b+c$ for all $c$. Moreover, $G^{+}=\{g \in G \mid g \geq 0\}$ is the positive cone of $G$. We shall use the references $[\mathrm{AF}],[\mathrm{BKW}],[\mathrm{D}]$, and [LZ] for various aspects of $\ell$-group theory. We sketch some particular ideas which we need here.

An ideal in an $\ell$-group is a convex sub- $\ell$-group; these are the kernels of $\ell$-homomorphisms. The collection of all ideals in the $\ell$-group $G$ is denoted $\operatorname{Idl}(G)$. Let $G$ be a sub- $\ell$-group of $H$; we write $G \leq H$. When the contraction $\operatorname{map} \operatorname{Idl}(H) \rightarrow \operatorname{Idl}(G)$ is one-to-one and onto, $H$ is called an a-extension of $G$, and we write $G \leq{ }_{a} H$.

Proposition 1.1 ([C1, 2.1]). $G \leq_{a} H$ if and only if $G \leq H$ and for each $h \in H^{+}$there is $g \in G^{+}$such that $g \sim_{a} h$, i.e., $h \leq m g$ and $g \leq n h$ for some positive integers $m, n$.

2000 Mathematics Subject Classification: 06F20, 54C40. 
If $G \leq_{a} H$ implies $G=H$, then $G$ is called $a$-closed. When $G \leq_{a} H$ and $H$ is $a$-closed, $H$ is called an $a$-closure of $G$. The first systematic study of $a$-extensions is Conrad's [C1]. A large literature has developed subsequently on this complicated subject; see especially $[\mathrm{AF}]$ and $[\mathrm{D}]$. Two main themes, which this paper continues, are: non-uniqueness of $a$-closure, even in quite restricted contexts; what does " $a$-closed" mean?

$\mathbb{N}, \mathbb{Z}, \mathbb{Q}$ and $\mathbb{R}$ denote the natural numbers, the integers, the rational numbers, and the reals, respectively, as one sort of mathematical structure or another, which being clear from context.

Let $G$ be an $\ell$-group, and $u \in G^{+}$. An element $g \in G$ is infinitesimal with respect to $h$, written $g \ll h$, if $0 \leq n g \leq h$ for all $n \in \mathbb{N}$. $G$ is archimedean if $g \ll h$ implies $g=0$. A positive element $u$ is a weak unit in $G$ if $|g| \wedge u=0$ implies $g=0$; and $u$ is a strong unit if $\langle u\rangle=G$, where $\langle u\rangle=\{g \in G \mid \exists n \in \mathbb{N}(|g| \leq n u)\}$ denotes the ideal in $G$ generated by $u$.

To illustrate: $\mathbb{R}$ is archimedean, and the class of archimedean $\ell$-groups is closed under product and sub- $\ell$-group formation. So, whenever $X$ is a topological space, which we always assume to be completely regular and Hausdorff (see $[\mathrm{GJ}]$ ), the $\ell$-group $C(X)$ of continuous $\mathbb{R}$-valued functions is archimedean. A function $u \in C(X)^{+}$is a weak unit if and only if $\operatorname{coz}(u)=$ $\{x \in X \mid u(x) \neq 0\}$ is dense in $X$; so the constant function 1 is a weak unit. For compact $X, 1$ is a strong unit since each $f \in C(X)$ is bounded.

The following is the classical first representation theorem of Yosida $[Y]$, elaborated and augmented somewhat. Many useful variations and generalizations are discussed in $[\mathrm{AF}],[\mathrm{LZ}],[\mathrm{HR}]$ and $[\mathrm{BH}]$.

THEOREM 1.2 (Representation of objects). Let $G$ be an archimedean $\ell$ group with strong unit $u$.

(a) The set of maximal ideals of $G$, with the hull-kernel topology, is a compact Hausdorff space, denoted $Y(G, u)$ or just $Y G$.

(b) There is an $\ell$-isomorphism $G \approx \widehat{G} \leq C(Y G)$ for which $\widehat{u}=1$ and $\widehat{G}$ separates the points of $Y G$.

(c) If $G \approx \widetilde{G} \leq C(X)$ is an $\ell$-isomorphism with $X$ compact Hausdorff and $\widetilde{u}=1$, then there is a continuous surjection $\tau: X \rightarrow Y G$ for which $\widetilde{g}=\widehat{g} \circ \tau$ for each $g \in G$. The group $\widetilde{G}$ separates points of $X$ if and only if $\tau$ is a homeomorphism.

(d) For each $p \in Y G, M_{p}=\{g \mid \widehat{g}(p)=0\}$ is a maximal ideal, and each maximal ideal is of this form for unique $p$. For each $p$, we have $\widehat{G} / M_{p} \approx\{\widehat{g}(p) \mid g \in G\} \leq \mathbb{R}$.

Theorem 1.3 (Representation of morphisms). Let $(G, u)$ and $(H, v)$ be archimedean $\ell$-groups with strong units $u, v$, and let $\varphi: G \rightarrow H$ be an $\ell$-homomorphism with $\varphi(u)=v$. Define a function $Y \varphi: Y G \leftarrow Y H$ by: $(Y \varphi)(p)=q$ means $\varphi^{-1}\left(M_{p}\right)=M_{q}$. Then $Y \varphi$ is continuous and is the 
unique map for which $\widehat{\varphi}(g)=\widehat{g} \circ(Y \varphi)$ for each $g \in G$. Further, $\varphi$ is oneto-one if and only if $Y \varphi$ is onto; if $\varphi$ is onto, then $Y \varphi$ is one-to-one.

Corollary 1.4. Suppose $G$ is archimedean with strong unit $u$, and $G \leq_{a} H$. Then $H$ is archimedean, $u$ is a strong unit in $H$, and $Y H=Y G$.

For the rest of the paper, we shall identify each archimedean $\ell$-group with strong unit with its Yosida representation: $1 \in G \leq C(Y G)$, and that notation means " $G$ is an archimedean $\ell$-group with strong unit in its Yosida representation".

For $X$ a topological space, $\operatorname{clop}(X)$ denotes the Boolean algebra of clopen subsets of $X$. For $U \subseteq X, \chi(U)$ denotes the characteristic function of $U$ and $g \chi(U)$ stands for the function on $Y G$ which vanishes off $U$ and agrees with $g$ on $U$. The expression " $g \chi(U) \in G$ " means there is $h \in G$ which in the Yosida representation is $g \chi(U)$. The following is simple but crucial.

Proposition 1.5. Suppose that $1 \in G \leq C(Y G)$. Then $g \chi(U) \in G$ for any $U \in \operatorname{cop}(Y G)$ and $g \in G$.

Proof. We first show that $\chi(U) \in G$ : Let $\{h<a\}$ stand for $\{x \in Y G \mid$ $h(x)<a\}$, and let $U^{\prime}=Y G-U$. For each $p \in U$ and each $q \in U^{\prime}$, choose $g_{p q} \in G$ which is 2 at $p$ and -1 at $q$. (From Theorem 1.2, there is $g \in G$ with $g(p)=1$ and $g(q)=0$. Let $g_{p q}=3 g-1$.) Fixing $p$, the set $\left\{\left\{g_{p q}<0\right\} \mid q \in U^{\prime}\right\}$ covers $U^{\prime}$, so for some finite $E \subseteq U^{\prime}$, the set $\left\{\left\{g_{p q}<0\right\} \mid q \in E\right\}$ covers $U^{\prime}$. Then $g_{p}=\left(\bigwedge_{q \in E} g_{p q}\right) \vee 0$ is 2 at $p$ and 0 on $U^{\prime}$. Now $\left\{\left\{g_{p}>1\right\} \mid p \in U\right\}$ covers $U$, so for some finite $F \subseteq U$, the set $\left\{\left\{g_{p}>1\right\} \mid p \in F\right\}$ covers $U$. Then $\chi(U)=\left(\bigvee_{p \in F} g_{p}\right) \wedge 1 \in G$.

If $g \geq 0$, there is $n$ with $g \leq n$, and then $g \chi(U)=g \wedge n \chi(U) \in G$. Finally, $g \chi(U)=(g \vee 0) \chi(U)-((-g) \vee 0) \chi(U) \in G$.

2. Hyperarchimedean $\ell$-groups with unit. A hyperarchimedean $\ell$ group is one for which each quotient is archimedean. We abbreviate "hyperarchimedean" to " $H A$ ". In this section we indicate some basic features of

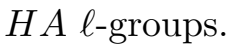

For $G$ an abelian $\ell$-group, $d G$ denotes the divisible hull. This is the unique divisible $\ell$-group containing $G$ for which $b \in d G$ implies there exists $a \in G$ for which $n b=a$ for some $n \in \mathbb{N}$. For $G$ archimedean with strong unit, viewed per Theorem 1.2 as $1 \in G \leq C(Y G)$, it is easily seen that $\{r g \mid g \in G, r \in \mathbb{Q}\} \leq C(Y G)$ is an explicit presentation of $d G$.

Proposition 2.1 ([C2]). (a) If $H$ is $H A$ and $G \leq H$, then $G$ is $H A$.

(b) If $G$ is $H A$ and $G \leq_{a} H$, then $H$ is $H A$.

(c) If $G$ is $H A$, then $d G$ is $H A$.

(d) If $G$ is $H A$, then any weak unit in $G$ is strong.

We turn to a discussion of the central role of "zero-sets". 
For $f \in \mathbb{R}^{X}$, the zero-set of $f$ is $Z(f)=f^{-1}(\{0\})$ and the cozero-set of $f$ is $\operatorname{coz}(f)=X-Z(f)$. If $f \in C(X)$, then $Z(f)$ is closed and $\operatorname{coz}(f)$ is open. For $1 \in G \leq C(Y G)$, let $\mathcal{Z} G=\{Z(g) \mid g \in G\}$; note that Theorem 1.3 says $\operatorname{clop}(Y G) \subseteq \mathcal{Z}(G)$. For $X$ a space, $S(X)=\{f \in C(X) \mid$ range $f$ is finite $\} \leq$ $C(X)$, and $S(X, \mathbb{Z})=\{f \in S(X) \mid$ range $f \subseteq \mathbb{Z}\} \leq S(X)$. Note that $\mathcal{Z} S(X)=\mathcal{Z} S(X, \mathbb{Z})=\operatorname{clop}(X)$.

Let $G$ be an $\ell$-group and $g \in G^{+}$. A component of $g$ is an $h \in G$ for which $h \wedge(g-h)=0$. The following is immediate.

Proposition 2.2. Let $1 \in G \leq C(Y G)$, and let $h \in G$. Then $h$ is a component of 1 if and only if there is $U \in \operatorname{clop}(Y G)$ for which $h=\chi(U)$.

In view of $[\mathrm{BKW}]$, Theorem 1.2 and Proposition 2.1 we obtain the following characterization of HA groups.

TheOREM 2.3. Let $G$ be an abelian $\ell$-group with weak unit $u$. The following are equivalent:

(a) $G$ is $H A$.

(b) $G$ is archimedean, $u$ is a strong unit, and in the Yosida representation $\mathcal{Z} G=\operatorname{clop}(Y G)$.

(c) $G$ is archimedean, $u$ is a strong unit, and for some representation $G \approx \widetilde{G} \leq C(X)$ with $X$ compact Hausdorff, $\widetilde{u}=1$, and $\mathcal{Z} \widetilde{G} \subseteq$ $\operatorname{clop}(X)$.

(d) $u$ is a strong unit, and for each $g \in G^{+}$there is a pair $(\chi, n)$, where $\chi$ is a component of $u$ and $n \in \mathbb{N}$, with $n g \geq \chi$ and $g \wedge(u-\chi)=0$.

(e) $G$ is archimedean, and in the Yosida representation $S(Y G, \mathbb{Z}) \leq_{a} G$.

Proposition 2.1, Theorem 2.3 and [C2] give the following.

Corollary 2.4. (a) If $G$ is $H A$ with strong unit, then $Y G$ is zerodimensional (i.e., $\operatorname{clop}(Y G)$ is a base for the open sets).

(b) For $X$ a space, $S(X)$ is $H A$.

(c) Let $G$ be an $\ell$-group. Then $G$ is $H A$ with strong unit if and only if there is a space $Y$ with $S(Y) \leq_{a} G$.

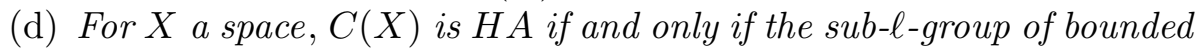
functions $C^{*}(X)$ is $H A$ if and only if $X$ is finite.

(e) If $G$ is $H A$ with strong unit, then: $G$ is a-closed if and only if $\left(G \leq_{a}\right.$ $H \leq C(Y G)$ implies $H=G)$.

Some of Theorem 2.3 generalizes as follows. First, if $G$ is archimedean with weak unit, in its Yosida representation (see [HR]), and $g \in G^{+}$then $Z(g)$ is open if and only if $g$ has a $(\chi, n)$ per Theorem 2.3(c). Second, for $G$ abelian with weak unit $u$, and $G^{*}=\langle u\rangle$, these are equivalent: $G$ is archimedean and $G^{*}$ is $H A ; G$ is archimedean and $\mathcal{Z} G=\operatorname{clop}(Y G)$; each 
$g \in G^{+}$has a $(\chi, n)$. These $\ell$-groups are called bounded away, and were introduced in the preprint $[\mathrm{KM}]$, and are discussed in [HKM].

This section concludes with the following useful results which show that if an $\ell$-group $G$ has $\ell$-group generators which seem hyperarchimedean, then $G$ actually is hyperarchimedean.

Let $H$ be an $\ell$-group, let $B$ be a subgroup of $H$, and let $\langle B\rangle$ denote the sub- $\ell$-group of $H$ generated by $B$. The elements of $\langle B\rangle$ are the elements of $H$ of the form $\bigwedge_{k} \bigvee_{j} b_{j k}$, for $b_{j k} \in B$ and finite index sets. (See [BKW].) The usual problem with analyzing $\langle B\rangle$ is the inscrutability of the expressions $\bigwedge \bigvee b_{j k}$.

Proposition 2.5. Suppose $X$ is compact and zero-dimensional, and that $B$ is a point-separating subgroup of $C(X)$ with $1 \in B$ and $Z(b)$ open for all $b \in B$. Then $\langle B\rangle$ is $H A$ and $\langle B\rangle=\left\{\sum_{i \in I} b_{i} \chi\left(U_{i}\right) \mid b_{i} \in B, U_{i} \in \operatorname{clop}(X)\right.$; I finite\}.

Corollary 2.6. Suppose $G$ is archimedean with strong unit: $1 \in G \leq$ $C(Y G)$, and suppose $b \in C(Y G)$ has $Z(g+z b)$ open for each $g \in G, z \in \mathbb{Z}$. Then

$$
\langle G+\mathbb{Z} \cdot b\rangle=G+\left\{\sum_{i \in I} z_{i} b \chi\left(U_{i}\right) \mid z_{i} \in \mathbb{Z}, U_{i} \in \operatorname{clop}(Y G) ; \text { I finite }\right\},
$$

$\langle G+\mathbb{Z} \cdot b\rangle$ is $H A$ and hence $G \leq_{a}\langle G+\mathbb{Z} \cdot b\rangle$. Moreover, if for each $U \in$

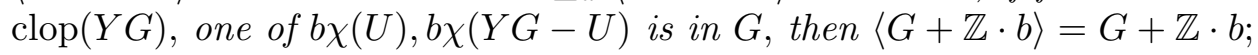
when $G$ is divisible, the converse holds.

The following lemma is necessary.

Lemma 2.7. If $I$ is finite and $h=\sum\left\{b_{i} \chi\left(U_{i}\right) \mid i \in I\right\}$, then for a finite set $\mathcal{E}$, there is a rewriting $h=\sum\left\{b_{E} \chi\left(V_{E}\right) \mid E \in \mathcal{E}\right\}$ with the sets $V_{E}$ disjoint and non-empty and $X=\bigcup\left\{V_{E} \mid E \in \mathcal{E}\right\}$.

Proof. For $E \subseteq I$, let $V_{E}=\bigcap\left\{U_{i} \mid i \in E\right\} \cap \bigcap\left\{X-U_{i} \mid i \notin E\right\}$, and let $\mathcal{E}=\left\{E \mid E \subseteq I, V_{E} \neq \emptyset\right\}$. Note that $V_{\emptyset}=X-\bigcup\left\{U_{i} \mid i \in E\right\}$. For $\emptyset \neq E \in \mathcal{E}$, let $b_{E}=\sum\left\{b_{i} \mid i \in E\right\}$, and set $b_{\emptyset}=0$. If $E \neq F$, say $i \in E$ but $i \notin F$, then $V_{E} \subseteq U_{i}$ while $V_{F} \subseteq X-U_{i}$, so $V_{E} \cap V_{F}=\emptyset$. For $p \in X$, let $E(p)=\left\{i \in I \mid p \in U_{i}\right\}$. Then $V_{E(p)} \neq \emptyset$, and so $E(p) \in \mathcal{E}$, and

$h(p)=\sum\left\{b_{i}(p) \mid i \in E(p)\right\}=b_{E(p)} \chi\left(V_{E(p)}\right)(p)=\sum\left\{b_{E} \chi\left(V_{E}\right) \mid E \in \mathcal{E}\right\}(p)$ since the $V_{E}$ 's are disjoint.

Proof of Proposition 2.5. Of course, $\langle B\rangle$ is archimedean with strong unit 1; by Theorem 1.2, $Y\langle B\rangle=X$ and the presentation of $\langle B\rangle$ is the Yosida representation. Let $B_{0}$ be the set of expressions $\sum b_{i} \chi\left(U_{i}\right)$. By Proposition 1.5, each $b \chi(u) \in\langle B\rangle$, so that $B_{0} \subseteq\langle B\rangle$, and $B_{0}$ clearly is a group. 
We shall show that $Z(h)$ is open for $h \in B_{0}$ and then that $B_{0}$ is an $\ell$-group. So $B_{0}=\langle B\rangle$, and by Theorem $2.3,\langle B\rangle$ is $H A$.

Now let $B_{0} \ni h=\sum b_{E} \chi\left(V_{E}\right)$ be written as in Lemma 2.7. Then $Z(h)=\bigcup\left\{Z(h) \cap V_{E}\right\}=\bigcup\left\{Z\left(b_{E}\right) \cap V_{E}\right\}$, by disjointness. Since each $Z\left(b_{E}\right)$ is supposed open, and each $V_{E}$ is open, $Z(h)$ is open.

For $B_{0}$ to be an $\ell$-group, it suffices that $h \in B_{0}$ imply $h \vee 0 \in B_{0}$ (see [BKW]). Again write $h=\sum b_{E} \chi\left(V_{E}\right)$ as in Lemma 2.7. Disjointness implies $h \vee 0=\sum\left(b_{E} \chi\left(V_{E}\right) \vee 0\right)$ and $b_{E} \chi\left(V_{E}\right) \vee 0=\left(b_{E} \vee 0\right) \chi(E)$. So $h \vee 0 \in B_{0}$.

Proof of Corollary 2.6. For the first part, just apply Proposition 2.5 to $B=G+\mathbb{Z} \cdot b$. For the second part: Let $U^{\prime}=Y G-U$. Suppose one of $b \chi(U), b \chi\left(U^{\prime}\right)$ is in $G$ for all $U \in \operatorname{clop}(Y G)$. For all $U, b \chi(U)=g+z \cdot b$ for some $g \in G, z \in \mathbb{Z}$. If $b \chi(U) \in G$, use $z=0$. If $b \chi\left(U^{\prime}\right) \in G$, then $B=b \chi(U)+b \chi\left(U^{\prime}\right)$, and we can use $z=-1$. This shows that any expression $g+\sum z_{i} b \chi\left(U_{i}\right)$ actually lies in $G+\mathbb{Z} \cdot b$. Conversely, suppose $\langle G+\mathbb{Z} \cdot b\rangle=$ $G+\mathbb{Z} \cdot b$, and $U \in \operatorname{clop}(Y G)$. Suppose $b \chi(U) \in G$, so $b \chi(U)=g+z b$ with $z \neq 0$. Then $b \chi(U)=g+z b=g \chi(U)+g \chi\left(U^{\prime}\right)+z b \chi(U)+z b \chi\left(U^{\prime}\right)$. For $x \in U^{\prime}$, this equation becomes $0=g(x)+z b(x)$. This shows $g \chi\left(U^{\prime}\right)+z b \chi\left(U^{\prime}\right)=0$. Since $G$ is divisible, $z \neq 0$, and $g \chi\left(U^{\prime}\right) \in G$ by Proposition 1.5, we have $b \chi\left(U^{\prime}\right) \in G$.

It is not difficult to construct an $H A$ group such that $\langle G+\mathbb{Z} \cdot b\rangle \neq G+\mathbb{Z} \cdot b$; we omit this.

3. The $P$-groups. $\alpha \mathbb{N}=\mathbb{N} \cup\{\alpha\}$ denotes the one-point compactification of the discrete space $\mathbb{N}$. In this section we describe all the groups $G$ with $S(\alpha \mathbb{N}, \mathbb{Z}) \leq_{a} G$. This is a generalization of

EXAMPLE 3.1. Let $C_{00}=\{f \in C(\alpha \mathbb{N}) \mid f$ vanishes on a neighborhood of $\alpha\}$. This is the weak product of countably many copies of $\mathbb{R}$, and $C_{00} \leq$ $S(\alpha \mathbb{N})$. Let $b(n)=\pi+1 / n$, so $b \in C(\alpha \mathbb{N})-S(\alpha \mathbb{N})$ and $b(\alpha)=\pi$. Write $\mathbb{R}=A \oplus \mathbb{Q} \pi$, as a direct sum of $\mathbb{Q}$-vector spaces.

(a) (P. Conrad $[\mathrm{C} 2,7.1])$ Let $J=\left(C_{00}+\mathbb{Q}\right)+\mathbb{Q} \cdot b$. Then $S(\alpha \mathbb{N}, \mathbb{Z}) \leq_{a}$ $J \nsubseteq S(\alpha \mathbb{N})$. Then $J$ is $H A$, cannot be represented as an $\ell$-group of step functions, and the vector lattice hull, $v J$, is not $H A$.

(b) (M. Anderson and P. Conrad $[\mathrm{AC}, 4.1])$ Let $K=\left(C_{00}+A\right)+\mathbb{Q} \cdot b$. Then $J \leq_{a} K$ and $K / M_{p}=\mathbb{R}$ for all $p \in \alpha \mathbb{N}$. So $K$ is $a$-closed, thus an $a$-closure of $J$. But $K$ is not a vector lattice. Note that, actually, [AC, p. 239] says " $K$ is the $a$-closure of $J$ ". It can be seen from Proposition 3.5 below that $J$ has $2^{\mathfrak{c}} a$-closures.

Some of these assertions about $J$ and $K$ are not particularly obvious. Some, not all, will be shown below. But we emphasize what is going on here: 
The signature of the hyperarchimedean situation, which is the basic theme of this paper, is the interplay between the locally constant functions and the non-locally constant functions. This takes a rather simple form for the groups in Example 3.1 above, and indeed for general $G$ with $Y G=\alpha \mathbb{N}$, as described in the rest of this section. For more general $G$, the interplay is highly visible, but more complicated and more difficult to describe. We quantify some of this at a rudimentary level:

For archimedean $G$ with strong unit and $Y G=\alpha \mathbb{N}$, let

$$
L(G, \alpha)=\{\delta \in \mathbb{R} \mid \exists g \in G(g=\delta \text { on a nbhd of } \alpha)\},
$$

$n L(G, \alpha)=\{\delta \in \mathbb{R} \mid \exists g \in G(g(\alpha)=\delta, g$ is not constant on any nbhd of $\alpha)\}$.

Regarding Example 3.1, we have

$$
\begin{aligned}
& J / M_{\alpha}=\mathbb{Q} \oplus \mathbb{Q} \cdot \pi, \quad L(J, \alpha)=\mathbb{Q}, \quad n L(J, \alpha)=J / M_{\alpha}-\mathbb{Q} ; \\
& K / M_{\alpha}=A \oplus \mathbb{Q} \cdot \pi=\mathbb{R}, \quad L(K, \alpha)=A, \quad n L(J, \alpha)=\mathbb{R}-A .
\end{aligned}
$$

Here we have $L(J, \alpha) \cap n L(J, \alpha)=\emptyset$, likewise for $K$, and this is why the groups are hyperarchimedean; and $K / M_{\alpha}=\mathbb{R}$, and this is why $K$ is $a$-closed. These ideas will be articulated fully for $Y G=\alpha \mathbb{N}$.

It will be helpful to keep these guidelines in mind as we proceed.

Suppose $S(\alpha \mathbb{N}, \mathbb{Z}) \leq S \leq S(\alpha \mathbb{N})$, let $F=S \cap C_{00}$ and $A=S / M_{\alpha}$. For each $s \in S$ and $n \in \mathbb{N}$, we have $s \chi(\{n\}) \in S$. Thus, $S / M_{n}=F / M_{n}$ for each $n \in \mathbb{N}$, for each $\delta \in A$ there is $n(\delta)$ such that $\delta \in F / M_{n}$ for $n \geq n(\delta)$, and $S=F+A$,

We prefer to start with $F$ and $A$ as initial data.

Proposition 3.2. Suppose $F \leq C_{00}, 1 \in A \leq \mathbb{R}$, and suppose that for each $\delta \in A$ there is $n(\delta)$ such that $\delta \in F / M_{n}$ for $n \geq n(\delta)$. Let $b \in C(\alpha \mathbb{N})-$ $S(\alpha \mathbb{N})$ with $b(n) \in F / M_{n}$ for each $n \in \mathbb{N}$. The following are equivalent:

(a) $b(\alpha) \notin d A=\mathbb{Q} \cdot A$.

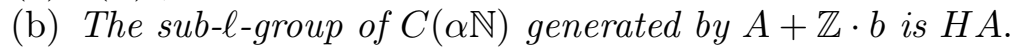

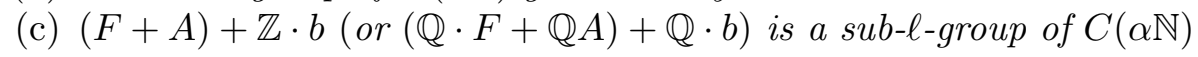
which is $H A$.

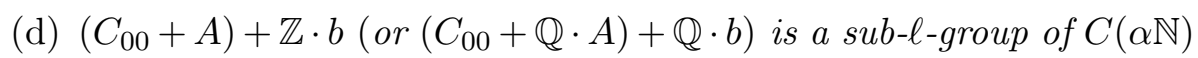
which is $H A$.

Proof. (a) $\Rightarrow(\mathrm{d})$. We use Corollary 2.6 with $G=C_{00}+A$. We first want to see that $Z(g+z b)$ is open for $g \in G$. This is obvious if $z=0$. So suppose $z \neq 0$. Note that $g(\alpha) \in A$. Thus $g(\alpha)+z b(\alpha) \neq 0$ by (a), so $Z(g+z b)$ is a finite subset of $\mathbb{N}$, thus open in $\alpha \mathbb{N}$. Now using the second part of Corollary 2.6, consider $b \chi(U)$ for $U \in \operatorname{clop}(\alpha \mathbb{N})$. Then $\alpha \notin U$ implies $U$ is finite, so $b \chi(U) \in C_{00} \subseteq G$, and $\alpha \in U$ implies $\alpha \mathbb{N}-U$ is finite, so $b \chi(\alpha \mathbb{N}-U) \in C_{00} \subseteq G$. The parenthetical part of (d) follows from Proposition 2.1(c). 
(d) $\Rightarrow(\mathrm{c}) \Rightarrow$ (b) follow by Proposition 2.1(a), using Proposition 2.1(c) for the parenthetical part of (c).

(b) $\Rightarrow$ (a). Let $G=\langle A+\mathbb{Z} \cdot b\rangle \leq C(\alpha \mathbb{N})$. By (b) and Theorem 2.3, $Z(\delta+z b)$ is open for each $z \in \mathbb{Z}$ and $\delta \in A$. As in (a) $\Rightarrow(\mathrm{d})$, this means that if $z \neq 0$, then $\delta+z b(\alpha) \neq 0$, which says (a).

Proposition 3.2 is a straightforward generalization of Example 3.1(a). We now use it to describe explicitly all the divisible $H A$ groups, $G$, with strong unit for which $Y G=\alpha \mathbb{N}$. The "divisible" restriction is simplifying, and from most points of view, without loss of generality.

So consider Proposition 3.2 supposing $F$ and $A$ are divisible, and let

$$
P=(F+A)+\mathbb{Q} \cdot b
$$

be the $H A$ l-group in Proposition 3.2(c). Then $P \cap C_{00}=F, P \cap S(\alpha \mathbb{N})=$ $F+A, P / M_{n}=F / M_{n}$ for each $n \in \mathbb{N}, P \cap S(\alpha \mathbb{N}) / M_{\alpha}=A$ and $P / M_{\alpha}=$ $A \oplus \mathbb{Q} \cdot b(\alpha)$ as a direct sum of $\mathbb{Q}$-vector spaces.

Construction 3.3. (a) Let $F$ and $A$ be as in Proposition 3.2 and divisible. Let $D \leq \mathbb{R}$ be divisible and $\mathbb{Q}$-linearly independent of $A$, i.e., $D \cap A=\{0\}$. Let $\varphi: D \rightarrow C(\alpha \mathbb{N})$ be a $\mathbb{Q}$-module homomorphism for which

(i) $\varphi(D) \cap S(\alpha \mathbb{N})=\{0\}$,

(ii) $\forall \delta \in D \forall n \in \mathbb{N}\left(\varphi(\delta)(n) \in F / M_{n}\right)$,

(iii) $\forall \delta \in D(\varphi(\delta)(\alpha)=\delta)$, which implies $\varphi$ is one-to-one.

Let $P(F, A,(D, \varphi))=(F+A)+\varphi(D)$.

(b) Before proceeding, we take note that plenty of these exist: Given $F, A, D$, let $D_{1}$ be a $\mathbb{Q}$-basis for $D$. Then let $\varphi_{1}: D_{1} \rightarrow C(\alpha \mathbb{N})-S(\alpha \mathbb{N})$ be "constructed" like this: $A$ and each $F / M_{n}$ are topologically dense in $\mathbb{R}$, so given $\delta \in D_{1}$, there is a sequence $a_{n} \in A$ with $\left|\delta-a_{n}\right|<1 / n$. Let $n_{1}$ be the first integer such that $\left(n>n_{1} \Rightarrow a_{1} \in F / M_{n}\right)$, let $n_{2}$ be the first integer $>n_{1}$ such that $\left(n>n_{2} \Rightarrow a_{2} \in F / M_{n}\right)$, and so on. Now let $f_{n}=1$ for $1 \leq n \leq n_{1}$, so these $f_{n} \in F / M_{n}$; let $f_{n}=a_{n}$ for $n_{1}<n \leq n_{2}$, so these $f_{n} \in F / M_{n}$, and so on.

Thus, $f_{n} \in F / M_{n}$ for all $n$, and $f_{n} \rightarrow \delta$. Define $\varphi_{1}(\delta)(n)=f_{n}$ and $\varphi_{1}(\delta)(\alpha)=\delta$, and now extend $\varphi_{1}$ to $\varphi: D \rightarrow C(\alpha \mathbb{N})$ by $\mathbb{Q}$-linearity. Clearly $\varphi$ satisfies (ii) and (iii) above. Easy examples show that the preceding precautions are necessary to ensure (i), which we now show: Since $\varphi$ is $1-1$, (i) just means that for all $\delta \in D-\{0\}, \varphi(\delta)$ is constant on no neighborhood of $\alpha$. But in fact there exists $n$ with $\varphi(\delta)(\alpha)=\delta$ if and only if $\delta=0$, since $\varphi(\delta)(n) \in A$ and $A$ and $D$ are $\mathbb{Q}$-linearly independent.

Theorem 3.4. (I) $P=P(F, A,(D, \varphi))$ is a divisible hyperarchimedean $\ell$-group with strong unit 1 and $Y P=\alpha \mathbb{N}$, with the features: 
$P \cap C_{00}=F$ and $P \cap S(\alpha \mathbb{N})=F+A$;

$P / M_{n}=F / M_{n}$ for all $n \in \mathbb{N}$ and $P \cap S(\alpha \mathbb{N}) / M_{\alpha}=L(P, \alpha)=A$;

$P / M_{\alpha}=A \oplus D$ and $n L(P, \alpha)=P / M_{\alpha}-A=(D-\{0\})+A$.

(II) If $G$ is any divisible hyperarchimedean $\ell$-group with strong unit, and $Y G=\alpha \mathbb{N}$, then there are $F, A, D, \varphi$ for which, in the Yosida representation of $G$, we have $G=P(F, A,(D, \varphi))$.

Note that in (II), given $G$, the sets $F$ and $A$ are determined by (I). But, by (I), $D$ only needs to have $A \oplus D=G / M_{\alpha}$, and situations $\left(D_{1} \neq D_{2}\right.$ with $\left.A \oplus D_{1}=A \oplus D_{2}\right)$ are common. Then of course, having fixed $D$, various $\varphi$ are possible. See below.

Proof. Note that $P(F, A,(D, \varphi))=\bigcup\{(F+A)+b \mid b \in \varphi(D)\}$.

(I) Clearly, $1 \in P \subseteq C(\alpha \mathbb{N})$ and $P$ separates the points of $\alpha \mathbb{N}$. If $0 \neq$ $b=\varphi(\delta)$ for some $\delta \in D$, then $\delta \neq 0$ and $\varphi(\delta)(\alpha) \notin A$, so Proposition 3.2(a) holds. Thus Proposition 3.2(c) holds, and $(F+A)+\mathbb{Q} \cdot b$ is an $H A$-group with Yosida space $\alpha \mathbb{N}$. Therefore, if $g_{i}=\left(f_{i}+a_{i}\right)+b_{i} \in P\left(i=1,2, b_{i} \in \varphi(D)\right.$, etc.) then $Z\left(g_{1}\right)$ is open by Theorem 2.3 , and $g_{1} \vee 0 \in(F+A)+\mathbb{Q} \cdot b_{1} \subseteq P$. Also, if $b_{i}=\varphi\left(\delta_{i}\right)$, then since $\varphi$ is $\mathbb{Q}$-linear, $b_{0}=b_{1}-b_{2}=\varphi\left(\delta_{1}-\delta_{2}\right)$, so that $g_{1}-g_{2} \in(F+A)+b_{0} \subseteq P$. So $P$ is an $\ell$-group with open zero-sets,

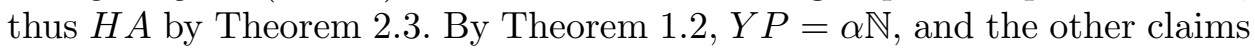
for $P$ are clear.

(II) Let $G$ be given, let $F=G \cap C_{00}$ and $A=G \cap S(\alpha \mathbb{N}) / M_{\alpha}=L(G, \alpha)$. Thus $n L(G, \alpha)=G / M_{\alpha}-A$.

Let $D_{1}$ be a subset of $G / M_{\alpha}$ maximal $\mathbb{Q}$-linearly independent of $A$, and let $\varphi_{1}: D_{1} \rightarrow G-S(\alpha \mathbb{N})$ be any choice function with $\varphi_{1}(\delta)(\alpha)=\delta$ for all $\delta \in D_{1}$. Let $D$ be the $\mathbb{Q}$-linear span of $D_{1}$ and let $\varphi: D \rightarrow G$ be the extension by $\mathbb{Q}$-linearity. This satisfies Construction 3.3(a)(i); i.e., the technicalities in Construction 3.3(b) are not needed here: If $\varphi(\delta) \neq 0$ then $0 \neq \delta=\varphi(\delta)(\alpha) \in G / M_{\alpha}-A$.

So let $P=P(F, A,(D, \varphi))$. Obviously, $P \subseteq G$. For the reverse, let $g \in G$. Our choice of $D$ makes $G / M_{\alpha}=A \oplus D$, so that $g(\alpha)=a+\delta$ for unique $a \in A, \delta \in D$. So there are $s \in G \cap S(\alpha \mathbb{N})$ and $b \in \varphi(D)$ with $a=s(\alpha)$ and $\delta=b(\alpha)$ and therefore $g(\alpha)=s(\alpha)+b(\alpha)$. Let $h=s+b \in(F+A)+b \subseteq P$. Since $h(\alpha)=g(\alpha)$, we have $h=g$ on a neighborhood $U$ of $\alpha$. Since $\alpha \mathbb{N}-U$ is finite, $g-h \in C_{00} \cap G=F$. Thus $g=(g-h)+h \in(F+A)+b \subseteq P$.

It is easy to show the following.

Proposition 3.5. Let $P_{i}=P\left(F_{i}, A_{i},\left(D_{i}, \varphi_{i}\right)\right)$ for $i=1,2$ be as in Construction 3.3 and Theorem 3.4. These are equivalent:

(a) $P_{1} \leq P_{2}$, so $P_{1} \leq{ }_{a} P_{2}$.

(b) $F_{1} \leq F_{2}, A_{1} \leq A_{2}$, and $P_{1} / M_{\alpha} \leq P_{2} / M_{\alpha}$. 
(c) $F_{1} \leq F_{2}, A_{1} \leq A_{2}$, and $P_{2}=P\left(F_{2}, A_{2},\left(D_{2}^{\prime}, \varphi_{2}^{\prime}\right)\right)$ for some $D_{2}^{\prime} \supseteq D_{1}$ and $\varphi_{2}^{\prime} \supseteq \varphi_{1}$.

Proposition 3.5 makes it obvious how to construct all $a$-extensions of an $\ell$-group $P=P(F, A,(D, \varphi))$ : enlarge $F, A$ and $D$ and then extend $\varphi$. When these are not possible, $P$ is $a$-closed:

Corollary 3.6. These are equivalent about $P=P(F, A,(D, \varphi))$ :

(a) $P$ is a-closed.

(b) $F=C_{00}$ and $P / M_{\alpha}=\mathbb{R}$.

(c) $F=C_{00}$ and for any $\mathbb{Q}$-bases $B$ for $A$ and $C$ for $D, B \cup C$ is a $\mathbb{Q}$-basis for $\mathbb{R}$, i.e., a Hamel basis.

Proof. (b) $\Leftrightarrow(\mathrm{c})$ since $P / M_{\alpha}=A \oplus D ;(\mathrm{a}) \Leftrightarrow(\mathrm{c})$ by inspection of Proposition 3.5 .

In Corollary 3.6, (b) $\Rightarrow(\mathrm{a})$ is a special case of a result from $[\mathrm{AC}] ;(\mathrm{a}) \Rightarrow(\mathrm{b})$ is also a consequence of topological properties of $\alpha \mathbb{N}$. Corollary 3.6 and Theorem 3.4(I) give the following.

Corollary 3.7. Let $A$ be a divisible subgroup of $\mathbb{R}$ with $1 \in A$, and let $D$ be any $\mathbb{Q}$-vector space complement of $A$ in $\mathbb{R}$. Then any $P=P\left(C_{00}, A\right.$, $(D, \varphi))$ is a-closed and $n L(P, \alpha)=\mathbb{R}-A$.

Question 3.8 ([C2, p. 217, open question 4]). Suppose that $G$ is a sub$\ell$-group of a product $H$ of reals which satisfies

(*) if $0<g \in G$, then $r<g<s$ for some $0<r, s \in \mathbb{R} \leq H$.

Must the sub-l-ring of $H$ generated by $G$ be $H A$ ?

In general, $(*)$ implies $G$ is $H A$. If $G$ has strong unit, $H A$ implies (*) for any such $G \leq H$, as is easily seen from Sections 1 and 2 here.

The answer to Question 3.8 is "No": Let $P=P\left(C_{00}, \mathbb{Q},(D, \varphi)\right)$ with $D=\mathbb{Q} \cdot \sqrt{2}$. Here $P \leq C(\alpha \mathbb{N}) \leq \mathbb{R}^{\mathbb{N} \cup\{\alpha\}}=H$, and the sub- $\ell$-ring of $H$ generated by $P$ is the sub- $\ell$-ring of $C(\alpha \mathbb{N})$ generated by $P$. Call this $\varrho P$. By Theorem 2.3, $\varrho P$ is $H A$ if and only if it has open zero-sets. But there is $b=\varphi(\sqrt{2}) \in P$ so $2-b^{2} \in \varrho P$, but $Z\left(2-b^{2}\right)$ is not open since $b^{2}$ cannot be 2 on a neighborhood of $\alpha$.

4. The $\Psi$-groups. As noted in the Abstract, the following progressively more pointed questions have been asked by Conrad, and Anderson and Conrad.

Question 4.1. Suppose that $G$ is a-closed.

(1) Is G/I a-closed for every ideal I?

(2) Is $G / P$ a-closed for every minimal prime $P$ ? 
(3) If $G$ is also $H A$, is $G / M$ a-closed for every maximal $M$, i.e., is $G / M=\mathbb{R}$ ?

Question (1) is from [C1, p. 153]; its converse is obviously true. Questions (2) and (3) are from [AC, p. 227]. The converse to (2) is Corollary I of [AC, p. 226], so the converse to (3) also holds.

We give the answer "No" to Question 4.1(3), so all these answers are "No", taking aim at the issue by analyzing, for (divisible) $H A \ell$-groups with strong unit $G$, the mechanics of $a$-extendibility in terms of permissible enlargements of the $G / M_{p}$, one point $p$ at a time. We make another construction to produce various $a$-closed $G$ for which various $G / M_{p}$ are various proper subgroups of $\mathbb{R}$.

Construction 4.2. Let $X$ be an index set, and $\left\{G_{x} \mid x \in X\right\}$ a set of archimedean $\ell$-groups with strong unit. Let $Y_{x}=Y G_{x}$ for $x \in X$, and let $Y=\alpha \sum\left\{Y_{x} \mid x \in X\right\}$ be the one-point compactification of the disjoint union: $Y=\{\alpha\} \cup \sum Y_{x}$, in which $U \subseteq \sum Y_{x}$ is open if and only if each $U \cap Y_{x}$ is open in $Y_{x}$, and every neighborhood of $\alpha$ contains all but finitely many $Y_{x}$. Let $1 \in A \leq \mathbb{R}$, construed as constant functions on $Y$, or on any $Y_{x}$, and suppose that $A \leq G_{x}$ for each $x$ for simplicity. $\Psi=\Psi\left(\left\{G_{x}\right\}, A\right)$ denotes the $\ell$-subgroup of $C(Y)$ generated by the weak product $\prod^{\omega} G_{x}$ and the constant functions from $A$.

It is easy to see that, for $f \in C(Y)$, we have $f \in \Psi$ if and only if there is a finite set $F \subseteq X$ and $a \in A$ such that $f \mid Y_{x} \in G_{x}$ for $x \in F$ and $f$ is constantly $a$ on $Y-\sum\left\{Y_{x} \mid x \in F\right\}$. Note that, for $g \in G_{x}$, the group $\Psi$ contains the function which is $g$ on $Y_{x}$ and 0 elsewhere; we denote this by $g \chi\left(Y_{x}\right)$. Clearly, for the divisible hull, $d \Psi=\Psi\left(\left\{d G_{x}\right\}, d A\right)$. Thus $\Psi=\prod^{\omega} G_{x}+A \leq C(Y)$ is archimedean with strong unit $1, Y \Psi=Y$ by Theorem 1.2(c), and evidently: for $p \in Y_{x}, \Psi / M_{p}=G_{x} / M_{p}$, and $\Psi / M_{\alpha}=A$. $\Psi\left(\left\{G_{x}\right\}, \mathbb{Z}\right)$ is the "unital version" of $\prod^{\omega} G_{x}$.

Note that the $\Psi$-groups are a partial generalization of the $P$-groups of $\S 3$ : $\Psi\left(\left\{\mathbb{R}_{n}\right\}, A\right)=P\left(\prod^{\omega} \mathbb{R}_{n}, A,(\emptyset, \emptyset)\right)$. We could complete the generalization by adding a $(D, \varphi)$ in the data for $\Psi$ and we shall do that if a purpose develops.

Recall that a function $f \in C(X)$ is locally constant at $p \in X$ if there is a neighborhood of $p$ on which $f$ is constant. In the following definition, $\delta$ is always a real number. Recall also that given $G$ and $p \in Y G$ we have $G / M_{p}=\{\delta \mid \exists g \in G(g(p)=\delta)\}$. We then define the analogous sets:

$$
\begin{aligned}
L(G, p) & =\{\delta \mid \exists g \in G \text { locally constant at } p(g(p)=\delta)\}, \\
n L(G, p) & =\{\delta \mid \exists g \in G \text { not locally constant at } p(g(p)=\delta)\} \\
n L(G) & =\bigcup\{n L(G, p) \mid p \in Y G\} .
\end{aligned}
$$

Observe that $n L(\Psi)=\bigcup\left\{n L\left(G_{x}\right) \mid x \in X\right\}$. 
We establish criteria for $\Psi$ to be $H A$.

Proposition 4.3. $\Psi\left(\left\{G_{x}\right\}, A\right)$ is $H A$ if and only if for each $x \in X$, $G_{x}$ is $H A$ and $n L\left(G_{x}\right) \cap A=\emptyset$.

Proof. Suppose $\Psi$ is $H A$. Restriction $\Psi \ni f \mapsto f \mid Y_{x} \in G_{x}$ is a surjective homomorphism, so $G_{x}$ is $H A$. This implies $n L\left(G_{x}\right) \cap A=\emptyset$ since $A \subseteq$ $L\left(G_{x}, p\right)$ for each $p \in Y_{x}$.

Conversely, if $g \in \Psi$, then $g=\sum\left\{g_{x} \chi\left(Y_{x}\right) \mid x \in F\right\}+a$ for a finite set $F$. If $a \neq 0$, then $Z(g)=\bigcup\left\{Z\left(g_{x}\right) \mid x \in F\right\}$ and this is open. If $a=0$, then $Z(g)=\bigcup\left\{Z\left(g_{x}\right) \mid x \in F\right\} \cup\left(\bigcup\left\{Y_{x} \mid x \notin F\right\}\right)$, which is open. By Corollary $2.6, \Psi$ is $H A$.

The following outlines properties of $a$-extensions of $\Psi$, when it is $H A$, based on the action of adjoining certain values. Let

$$
\begin{aligned}
A d(G, p) & =\left\{\delta \mid \delta \notin G / M_{p}, \exists G \leq_{a} H\left(\delta \in H / M_{p}\right)\right\}, \\
A d L(G, p) & =\left\{\delta \mid \delta \notin G / M_{p}, \exists G \leq_{a} H(\delta \in L(H, p))\right\}, \\
A d n L(G, p) & =\left\{\delta \mid \delta \in G / M_{p}, \exists G \leq_{a} H(\delta \in n L(H, p))\right\} .
\end{aligned}
$$

We now examine $\operatorname{Ad}(G, p)=A d L(G, p) \cup \operatorname{Adn} L(G, p)$, by examining the pieces separately.

THEOREM 4.4. Let $p \in Y G$, and let $\delta$ be a real number such that $\delta \notin$ $G / M_{p}$. These are equivalent:

(a) $\delta \in A d L(G, p)$.

(b) There is $U \in \operatorname{clop}(Y G)$ with $p \in U$, and there is $G \leq_{a} H$ with $\delta \chi(U) \in H$.

(c) There is $U \in \operatorname{cop}(Y G)$ with $p \in U$ for which $\delta \notin n L(G, x)$ for each $x \in U$, i.e., $U \cap\{x \mid \delta \in n L(G, x)\}=\emptyset$.

Proof. $(\mathrm{a}) \Leftrightarrow(\mathrm{b})$ follows by noticing that, via Corollary 1.4, we get: $\delta \in$ $L(G, p)$ if and only if there is $U \in \operatorname{clop}(Y G)$ with $p \in U$, and $\delta \chi(U) \in G$.

(a) $\Rightarrow$ (c). If $G \leq_{a} H$ and $\delta \in L(H, p)$, then $\delta \notin n L(H, p) \supseteq n L(G, p)$ by the note above.

(c) $\Rightarrow$ (b). Assuming (b), let $H=\langle G+\mathbb{Z} \cdot \delta \chi(U)\rangle$. We use Corollary 2.6 to see that $G \leq_{a} H$. Consider $Z=Z(g+z \delta \chi(U))$. We have $Z=(Z \cap$ $U) \cup(Z \cap(Y G-U))$, and we show that each piece is open. The second, $Z \cap(Y G-U)=Z(g) \cap(Y G-U)$, is open since $Z(g)$ is open since $G$ is $H A$, and $U$ is closed. Now $Z \cap U=\{x \in U \mid g(x)=-z \delta\}$. So, if $x \in Z \cap U$, then $g$ is locally constant at $x$ since $\delta \notin n L(G, x)$ and $G$ is divisible, and there a is clopen $V \ni x$ with $g=-z \delta$ on $V$, so $V \subseteq Z \cap U$. Thus $Z \cap U$ is open.

Lemma 4.5. Suppose $\Psi=\Psi\left(\left\{G_{x}\right\}, A\right)$ is $H A$. If $\Psi \leq_{a} H$, then for each $x, G_{x} \leq_{a} H \mid Y_{x}$. Conversely, if $G_{x} \leq_{a} H_{x}$ for each $x$, then $\Psi\left(\left\{G_{x}\right\}, A\right)$ $\leq_{a} \Psi\left(\left\{H_{x}\right\}, A\right)$. 
Proof. The notation makes sense since $Y H_{x}=Y G_{x}$ for each $x$. The first assertion is because $G \leq_{a} H$ implies $G / I \cap G \leq_{a} H / I$ for each ideal $I$. Conversely, if $h=\sum\left\{h_{x} \chi\left(Y_{x}\right) \mid x \in F\right\}+a$, and for each $x \in F$ we have $g_{x} \sim_{a} h_{x}$, then $\sum\left\{g_{x}\left|\chi\left(Y_{x}\right)\right| x \in F\right\} \sim_{a} h$.

Proposition 4.6. (a) Let $G$ be $H A$. Then $G$ is a-closed if and only if $A d(G, p)=\emptyset$ for each $p \in Y G$.

(b) Suppose $\Psi=\Psi\left(\left\{G_{x}\right\}, A\right)$ is $H A$. Then $\Psi$ is a-closed if and only if each $G_{x}$ is a-closed and $\operatorname{Ad}(\Psi, \alpha)=\emptyset$.

Proof. (a) " $\Rightarrow$ " is clear. Conversely, let $G \leq_{a} H$ and $h \in H$. Since all $A d(G, p)$ are empty, for each $p$ there is $g_{p} \in G$ with $g_{p}(p)=h(p)$. Since $H$ is $H A, h-g_{p}$ is constant on a clopen $U_{p}$ containing $p$, which means $h \chi\left(U_{p}\right)=g \chi\left(U_{p}\right) \in G$, by Theorem 1.3. By compactness, there is a finite $F$ with $\bigcup\left\{U_{p} \mid p \in F\right\}=Y G$. Then $h=\bigvee_{p \in F}$ and $g \chi\left(U_{p}\right) \in G$.

(b) By (a), $\Psi$ is a-closed if and only if $A d(\Psi, p)=\emptyset$ for each $p \in Y$. For $p \in Y_{x}$, we have $A d(\Psi, p)=A d\left(G_{x}, p\right)$ using Lemma 4.5. The result follows.

We need the following well known lemma:

Lemma 4.7. $X$ be compact zero-dimensional. For $Z \subseteq X$, the following are equivalent: $Z \in \mathcal{Z} C(X) ; Z$ is a closed $G_{\delta}$-set; $Z$ is a countable intersection of clopen sets.

Theorem 4.8. Given $G, p \in Y G$, and real $\delta \notin G / M_{p}$ :

(I) If $\delta \in \operatorname{Adn} L(G, p)$, then there is a zero-set $Z$ with $p \in \partial Z$ with int $Z \cap\{x \mid \delta \in n L(G, x)\}=\emptyset$ and $\partial Z \cap\{x \mid \delta \in L(G, x)\}=\emptyset$.

(II) If there is a zero-set $Z$ with $p \in \partial Z$ with $\operatorname{int} Z \cap\{x \mid \delta \in n L(G, x)\}$ $=\emptyset$ and $\partial Z \cap\left\{x \mid \delta \in G / M_{x}\right\}=\emptyset$, then $\delta \in \operatorname{AdnL}(G, p)$.

Proof. (I) Suppose $\delta \in \operatorname{AdnL}(G, p)$, so $\delta \notin G / M_{p}$ and there is $G \leq_{a} H$ with $h \in H$ for which $h(p)=\delta$ and $h$ is not locally constant at $p$. Let $Z=\{x \mid h(x)=\delta\}$. This is a zero-set and clearly for $x \in Z$ :

(a) $x \in \operatorname{int} Z$ if and only if $h$ is locally constant at $x$ and then $\delta \in L(H, x)$;

(b) $x \in \partial Z$ if and only if $h$ is not locally constant at $x$ and then $\delta \in$ $n L(H, x)$.

By (b), $p \in \partial Z$. Since $n L(G, x) \subseteq n L(H, x)$ and $n L(H, x) \cap L(H, x)=\emptyset$, we see that int $Z \cap\{x \mid \delta \in n L(G, x)\}=\emptyset$ from (a). Since $L(G, x) \subseteq L(H, x)$ and $n L(H, x) \cap L(H, x)=\emptyset$, we get $\partial Z \cap\{x \mid \delta \in L(G, x)\}=\emptyset$ from (b).

(II) Let $Z$ be as described. By Lemma 4.7, $Y G-Z=\bigcup_{n} U_{n}$ for $U_{n} \in$ $\operatorname{clop}(Y G)$. Let $K_{n}=U_{n}-\bigcup_{i<n} U_{i}$. These are disjoint and clopen, $\bigcup K_{n}=$ $Y G-Z$ and $Y G=\bigcup K_{n} \cup Z$. Let $\left(r_{n}\right)$ be a sequence of rational numbers with $r_{n} \rightarrow \delta$. Define $b \in C(Y G)$ as $b(x)=r_{n}$ for $x \in K_{n}$ and $b(x)=\delta$ for $x \in Z$. Then $Z=\{x \mid b(x)=\delta\}$, and again for $x \in Z$ : 
(a) $x \in \operatorname{int} Z$ if and only if $b$ is locally constant at $x$;

(b) $x \in \partial Z$ if and only if $b$ is not locally constant at $x$.

Let $H=\langle G+\mathbb{Z} \cdot b\rangle$. We shall use Corollary 2.6 to show that $H$ is $H A$ so that $G \leq_{a} H$. It follows that $\delta \in n L(H, p)$, so $\delta \in \operatorname{Adn} L(G, p)$.

Let $g \in G$ and $z \in \mathbb{Z}$. We show that $E=Z(g+z b)$ is open. Now $E=E_{1} \cup E_{2} \cup E_{3}$, where $E_{1}=\bigcup\left(K_{n} \cup E\right), E_{2}=\operatorname{int} Z \cap E$, and $E_{3}=$ $\partial Z \cap E$. The set $E_{1}$ is open since $K_{n} \cap E=K_{n} \cap\left\{x \mid g(x)=-z r_{n}\right\}$ is open. $E_{2}$ is open: since int $Z \cap\{x \mid \delta \in n L(G, x)\}=\emptyset$, it is also the case that int $Z \cap\{x \mid-z \delta \in n L(G, x)\}=\emptyset$ since $G$ is divisible. Thus, if $x \in E_{2}$ then there is a neighborhood $V$ of $x$ on which $g=-z \delta$ and $x \in V \cap \operatorname{int} Z \subseteq E_{2}$. Finally, $E_{3}=\emptyset$ since $\partial Z \cap\left\{x \mid \delta \in G / M_{x}\right\}=\emptyset$ and hence $\partial Z \cap\left\{x \mid-z \delta \in G / M_{x}\right\}=\emptyset$ since $G$ is divisible.

It is not difficult to construct examples illustrating the gap between the conditions in Theorem 4.8(I) and (II). We omit this.

So we can focus on $A d(\Psi, \alpha)$. Keeping Theorem 4.4 in mind, note that if $\alpha \in U \subseteq Y$, then $U$ contains a clopen set containing $\alpha$ if and only if there is a finite $F$ such that $U \supseteq \bigcup\left\{Y_{x} \mid x \notin F\right\} ; U$ contains a zero-set containing $\alpha$ if and only if there is countable $F$ with $U \supseteq \bigcup\left\{Y_{x} \mid x \notin F\right\}$ by Lemma 4.7.

Proposition 4.9. Suppose $\Psi=\Psi\left(\left\{G_{x}\right\}, A\right)$ is $H A$ with $X$ uncountable.

(a) $\delta \in A d L(\Psi, \alpha)$ if and only if there is finite a $F \subseteq X$ for which $x \notin F$ implies $\delta \notin n L\left(G_{x}\right)$.

(b) $\delta \in \operatorname{AdnL}(\Psi, \alpha)$ if and only if there is a countable $F \subseteq X$ for which $x \notin F$ implies $\delta \notin n L\left(G_{x}\right)$.

Proof. (a) $n L(\Psi, \alpha)=\emptyset$ and for $p \in Y_{x}, n L(\Psi, p)=n L\left(G_{x}, p\right)$. So the condition says that $U=\bigcup\left\{Y_{x} \mid x \notin F\right\}$ satisfies Theorem 4.4(c).

(b) We make the following obvious, but useful, observation: If $G$ is $H A$ and $T \subseteq Y G$ is closed, then int $T \cap\{x \mid \delta \in n L(G, x)\}=\emptyset$ if and only if $T \cap\{x \mid \delta \in n L(G, x)\} \subseteq \partial T$ and $\partial T \cap\{x \mid \delta \in L(G, x)\}=\emptyset$ if and only if $T \cap\{x \mid \delta \in L(G, x)\} \subseteq \operatorname{int} T$.

Consider this observation for $Z$ a zero-set of $Y$ containing $\alpha$. Here $\partial Z=$ $\{\alpha\}, L(\Psi, \alpha)=A$ and $n L(\Psi, \alpha)=\emptyset$. So for $\delta \notin A$, we see that $\partial Z \cap\{x \mid \delta \in$ $\left.\Psi / M_{x}\right\}=\emptyset$, thus $\partial Z \cap\{x \mid \delta \in n L(\Psi, x)\}=\emptyset$ and the conditions in the two parts of the observation each reduce to: int $Z \cap\{x \mid \delta \in n L(\Psi, x)\}=\emptyset$. The condition in (b) says that for $Z_{0}=\bigcup\left\{Y_{x} \mid x \notin F\right\}$ we have $Z_{0} \cap\{x \mid \delta \in$ $n L(\Psi, x)\}=\emptyset$. Now apply Theorem 4.8 .

Corollary 4.10. Let $\Psi=\Psi\left(\left\{G_{x}\right\}, A\right)$ be $H A$ with $X$ uncountable. Then $A d(\Psi, \alpha)=\emptyset$ if and only if for each $\delta \notin A[$ for each countable $F \subseteq X$, there is $x \notin F$ with $\delta \in n L\left(G_{x}\right)$ ]. 
Proof. The condition bracketed is the negation of the condition in Proposition 4.9(b), which implies the negation of the condition in Proposition $4.9(\mathrm{a})$.

Corollary 4.11. Let $G_{0}$ be $H A$, let $X$ be uncountable and for each $x \in X$ let $G_{x}=G_{0}$. Let $\Psi=\Psi\left(\left\{G_{x}\right\}, A\right)$. Then $\Psi$ is $H A$ and $A d(\Psi, \alpha)=\emptyset$ if and only if $\mathbb{R}-A=n L\left(G_{0}\right)$.

Such $G_{0}$ are given in Corollary 3.7.

EXAMPLE 4.12. Let $A$ be any divisible subgroup of $\mathbb{R}$ containing 1 . There is an $a$-closed $\Psi_{A}$ that is $H A$ with strong unit, with a maximal ideal $M$ for which $\Psi_{A} / M=A$.

Such a $\Psi_{A}$ is a $\Psi$ in Corollary 4.11 , with $M=M_{\alpha}$ using $G_{0}=P\left(\left\{C_{00}\right\}\right.$, $A,(D, \varphi)) \leq C(\alpha \mathbb{N})$ from $\S 3$, with a $\varphi$ for which $A \oplus D=\mathbb{R}$. Now $G_{0}$ is $a$-closed by Corollary 3.6, so $\Psi_{A}$ is $a$-closed by Proposition $4.6(\mathrm{~b})$ and Corollary 4.11. As noted in Corollary 3.7, $n L\left(G_{0}, \alpha_{0}\right)=\mathbb{R}-A$ (writing $\alpha \mathbb{N}=\mathbb{N} \cup\left\{\alpha_{0}\right\}$ to avoid confusion).

For $\Psi_{A}$ in Example $4.12, \Psi_{A} / M_{p}=\mathbb{R}$ for every $p \neq \alpha$, and $\Psi_{A} / M_{\alpha}=A$. Of course, more complicated situations can be constructed. We content ourselves with just one more level of complexity.

ExAmple 4.13. Let $A$ be any divisible subgroup of $\mathbb{R}$ with $1 \in A$ and let $m$ be any uncountable cardinal number. Let $\alpha D(m)$ be the one-point compactification of the discrete space of cardinal $m$. There is an $a$-closed $\Psi=\Psi_{A, m}$, that is $H A$ with strong unit, for which $Y \Psi$ contains a copy of $\alpha D(m)$ as a nowhere dense subset with $\Psi / M_{p}=A$ for each $p \in \alpha D(m)$ :

Let $\Psi=\Psi\left(\left\{H_{i} \mid i<m\right\}, A\right)$ with each $H_{i}$ given by the $\Psi_{A}$ of Example 4.12. Here, $n L\left(H_{i}\right)=\mathbb{R}-A$ and since $n L\left(\Psi_{A}\right)=\mathbb{R}-A$, we see that $\Psi$ is $a$-closed by Proposition 4.6(b) and Corollary 4.10. We have $Y \Psi=$ $\{\alpha\} \cup \sum Y H_{i}$ and $\Psi / M_{\alpha}=A$. Also $Y H_{i}=\left\{\alpha_{i}\right\} \cup \sum(\cdot)$ and $H_{i} / M_{\alpha_{i}}=A$. The desired copy of $\alpha D(m)$ is $\{\alpha\} \cup\left\{\alpha_{i} \mid i<m\right\}$.

With reference to "nowhere dense", it is easy to show that: there is an $a$-closed $G$ for which $\left\{p \in Y G \mid G / M_{p} \neq \mathbb{R}\right\}$ has interior if and only if there is $a$-closed $H$ for which $\left\{p \in Y H \mid H / M_{p}=\mathbb{R}\right\}=\emptyset$ where $G$ and $H$ are $H A$ with strong unit. We do not know if such $G, H$ exist.

\section{References}

[AC] M. Anderson and P. Conrad, Epicomplete $\ell$-groups, Algebra Universalis 12 (1981), 224-241.

[AF] M. Anderson and T. Feil, Lattice-Ordered Groups, Reidel, Dordrecht, 1989. 
[BH] R. N. Ball and A. W. Hager, Applications of spaces with filters to archimedean

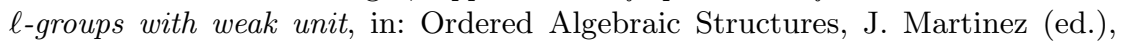
Kluwer, Dordrecht, 1989, 99-112.

[BKW] A. Bigard, K. Keimel et S. Wolfenstein, Groupes et Anneaux Réticulés, SpringerVerlag, Berlin, 1977.

[C1] P. Conrad, Archimedean extensions of lattice-ordered groups, J. Indian Math. Soc., 30 (1966), 131-160.

[C2] - Epi-archimedean groups, Czechoslovak Math. J. 24 (1974), 192-218.

[D] M. Darnel, Theory of Lattice-Ordered Groups, Dekker, New York, 1995.

[GJ] L. Gillman and M. Jerison, Rings of Continuous Functions, Van Nostrand, Princeton, 1960; reprinted as Grad. Texts in Math. 43, Springer-Verlag, New York, 1976.

[HKM] A. W. Hager, C. M. Kimber and W. W. McGovern, Least integer closed groups, in: Ordered Algebraic Structures, J. Martinez (ed.), Kluwer, Dordrecht, 2002, 245-260.

[HR] A. W. Hager and L. C. Robertson, Representing and ringifying a Riesz space, in: Sympos. Math. 21, Academic Press, London, 1977, 411-431.

[KM] C. Kimber and W. McGovern, Bounded away lattice-ordered groups, manuscript, 1998.

[LZ] W. Luxemburg and A. Zaanen, Riesz Spaces I, North-Holland, Amsterdam, 1971.

[Y] K. Yosida, On the representation of the vector lattice, Proc. Imp. Acad. Tokyo 18 (1942), 339-343.

Department of Mathematics and Computer Science

Wesleyan University

Department of Mathematics

Middletown, CT 06459, U.S.A.

Lafayette College

E-mail: ahager@wesleyan.edu

Easton, PA 18042, U.S.A.

E-mail: kimberc@lafayette.edu

Received 7 March 2002;

in revised form 24 February 2004 